\title{
Preface
}

\section{ABC Transporters}

ATP-binding cassette (ABC) transporters form a large superfamily of active transport systems found in archaea, eubacteria, and eukaryotes. They typically consist of two integral membrane domains containing six transmembrane $\alpha$-helices forming the translocation path, and two cytosolic nucleotide-binding domains that couple ATP binding to the translocation of the substrate. In bacteria, ABC transport systems are often half-size transporters having only one transmembrane domain fused to one nucleotide-binding domain. They probably dimerise to form a full-size transporter to mediate mainly the influx of essential compounds. Eukaryotic ABC transporters, on the other hand, function as exporters mediating the efflux of compounds from the cytosol to the extracellular space or to the inside of intracellular compartments, i.e., endoplasmic reticulum, mitochondria, peroxisomes, or vacuoles.

In this special issue, 21 expert reviews convey the excitement in the field. The papers illustrate the amazing progress in understanding the structural and functional aspects as well as the substantial achievements in the molecular characterization of $\mathrm{ABC}$ transporters from bacteria, yeast, mammals, and plants. This special issue accompanies the forthcoming FEBS Special Meeting "ATP-Binding Cassette (ABC) Proteins: From Multidrug Resistance to Genetic Diseases" in Innsbruck, Austria, the fifth in a highly successful series of conferences, which aims at being a worldwide forum for discussing progress in basic and applied $\mathrm{ABC}$ transporter research.

We would like to thank our colleagues for their time and efforts in making this a highly informative and stimulating issue and Patricia McCabe, the assistant manager editor of FEBS Letters, for her continuous support during the preparation of this special issue.

Ulf-Ingo Flügge

Gerrit van Meer

E-mail addresses: ui.fluegge@uni-koeln.de (U.-I. Flügge), g.vanmeer@chem.uu.nl (G. van Meer) 